Research Article

\title{
Swing Characteristics and Vibration Feature of Tower Cranes under Compound Working Condition
}

\author{
Fu Liu, ${ }^{1}$ Jianwei Yang $\mathbb{D}^{1,2}$ Jinhai Wang, ${ }^{1}$ and Changdong Liu ${ }^{1}$ \\ ${ }^{1}$ School of Mechanical-electronic and Vehicle Engineering, Beijing University of Civil Engineering and Architecture, \\ Beijing 100044, China \\ ${ }^{2}$ Beijing Engineering Research Center of Monitoring for Construction Safety, Beijing 100044, China \\ Correspondence should be addressed to Jianwei Yang; yangjianwei@bucea.edu.cn
}

Received 4 September 2021; Accepted 24 November 2021; Published 21 December 2021

Academic Editor: Letícia Fleck Fadel Miguel

Copyright (c) $2021 \mathrm{Fu}$ Liu et al. This is an open access article distributed under the Creative Commons Attribution License, which permits unrestricted use, distribution, and reproduction in any medium, provided the original work is properly cited.

\begin{abstract}
The swing behaviour of tower cranes under compound working conditions is closely related to construction safety and structural health. This paper presents dynamic models and simulated them for parameter analysis to understand tower cranes' dynamic characteristics and vibration features under compound working conditions. The parameters contain payload mass, rope length, lifting acceleration, slewing acceleration, luffing acceleration, and initial angle. For the lifting-luffing coupling motion (LLCM) and lifting-slewing coupling motion (LSCM) of the tower crane, the D'Alembert principle provides a theoretical basis for the derivation of system dynamics equations. The spatial swing angle description of the crane payload includes the time-domain response and frequency-domain response, which uses a dynamic model. The result shows that the mass has little effect on the spatial swing angle. The value of the lifting acceleration is stable at $0.004 \mathrm{~m} / \mathrm{s}^{2}$ to $0.01 \mathrm{~m} / \mathrm{s}^{2}$. Peak value (PV), root mean square value (RMS), root mean square frequency (RMSF), and frequency standard deviation (RVF) present the best sensitivity to changes in the spatial swing angle response. When PV of angles $\theta$ and $\beta$ increases by tens of thousands of times in the LLCM, PV can reflect the phenomenon of angle divergence. The skewness value (SV) increases by $3422 \%$ at the severe swing angle performance in the LSCM. The swing angle regularity with the compound working conditions can provide theoretical guidance for eliminating structural vibration.
\end{abstract}

\section{Introduction}

Tower cranes are essential equipment for large and heavy objects transportation in many industries. Although endless new products have created improvements in efficiency and reliability, their basic structure and technology have not changed. The large-scale, rapid, and modular development of tower cranes has brought considerable challenges to construction safety under compound working conditions. It may increase the shaking of the tower crane, which is not conducive to the positioning of the tower crane and brings safety hazards to goods and staff. Therefore, it is necessary to investigate the vibration features of the crane payload with the compound working conditions to provide practical guidance for applying vibration elimination techniques.
The research of tower cranes mainly focuses on the mechanical properties of the structural components, the response of the swing angle, and the performance control under some working conditions. In addition, many studies consider the only slewing, only lifting, or only luffing motion that produces the spatial motion of the pendulum. The study also obtained the dynamic performance of the mechanical moment under the acceleration and the deceleration of the slewing movement [1]. Yao et al. studied the dynamic process of a dual-trolley overhead crane transshipping rigidbody cargo with degrees of spatial freedom [2]. The flexible model of crane luffing motion is established, indicating that the crane's dynamic responses are more sensitive to the luffing acceleration than the luffing velocity [3]. The study found that the horizontal inertial forces in the radial direction are of no less importance than the forces in the 
tangential direction in the slewing motion [4]. Neupert et al. set up the mobile port crane's dynamic swing model during the luffing motion [5]. Feng et al. investigated the influence of dynamic parameters such as the velocity and acceleration of the trolley motion, payload mass, cable length on jib vibration, and payload swing during the luffing motion [6]. Jerman and Hribar drew the horizontal inertial forces factor of a swinging payload mass during the slewing motion [7]. Augustaitis et al. used the original software package to develop the model of the lift mechanism and then found the dynamic load during the lifting motion [8]. By calculating tipping loads at various load radius for a particular boom length, Shaikh and Kumar analyzed the stability factor during the luffing motion [9]. Trabka developed the numerically efficient telescopic crane model with the use of the finite element method during the luffing motion [10].

Many papers have studied the motion of the pendulum angle and related parameters. Some studies [11-13] investigated carrying pendulum systems and assumed these systems as a crane moving in the horizontal plane. De Angelis derived an observation model from the equation of motion of the coupled slung load system. The analysis of the swing angle plays a vital role in the antisway methods [14]. Ebrahimi et al. developed a method to estimate the swing angle using load cell sensors [15]. The performance evaluation experiment used a laboratory tower crane with cable length variations and under simultaneous tangential and radial crane motions [16]. Fatehi et al. developed a dynamic model that includes both the flexible cable's transverse vibrations and large swing angles while the trolley moves horizontally [17]. Fatehi et al. proposed a second-order singular perturbation formulation. The formulation can divide the crane dynamics into two onedegree actuated fast and slow subsystems [18]. Chin et al. modelled a boom crane as a spherical pendulum and a rigid system with two degrees of freedom. The research assumes that the influence comes from the platform motion [19]. Oguamanam et al. studied the dynamic of the overhead crane system, which uses a beam model to display the flexibility of the crane structure [20]. Also, Ghigliazza and Holmes regarded the crane structure as rigid bodies with discrete springs [21]. Ju and Choo considered the tower crane system's natural vibration and dynamic response due to the acceleration or deceleration of the payload. The study involved the vertical direction of the payload movement [22]. Gasic et al. discussed the two-dimensional inertial effects of the trolley, hoist, and payload masses [23]. Another study developed a mathematical model of crawler cranes for heavy tasks. It activated the payload-lifting and boom-hoisting motions simultaneously [24]. Liu et al. found that increasing the mass of the payloads leads to a larger deflection of the beam [25]. Marinovic et al. presented a multimass model of a slewing crane, which considered the crane's slewing motion that produces the payload's spatial oscillation [26].

According to the model of the underactuated tower crane swing angle, most studies investigated combine the feedforward compensation and online estimation. A robust control technique based on adaptive fuzzy control is investigated for trajectory tracking of multi-input multioutput (MIMO) underactuated system, subject not only to system uncertainties but also to the unknown actuator deadzone band nonlinearities in the motors of the MIMO underactuated system [27]. The feedforward solution is made robust by an augmentation with LQR as well as a sliding mode controller [28]. This article presents an output feedback controller, which can simultaneously solve the problems of saturation constraints and velocity signal unavailability [29]. A new model-free robust control scheme for payload swing angle attenuation of two-dimensional crane systems with varying rope length is introduced in this work [30].

Many research studies only have established and analyzed a slewing condition model, a lifting condition model, a luffing condition model, or a slewing-luffing compound condition model of the tower crane. Notably, tower cranes have worked for a long time under LLCMs and LSCMs. However, the current research rarely conducts quantitative analysis under these two conditions.

This paper introduces two mathematical models by D'Alembert's principle and corresponding model verification. After that, the following sections illustrate angular response and then discuss the simulation results. The final section proposes the main conclusion.

\section{Dynamics System and Model}

2.1. Lifting-Luffing Motion Model. Figure 1 shows the schematic diagram of lifting and luffing under the compound working conditions. There is an orthogonal coordinate system in the model. The space model of the liftingluffing condition is projected to the $x-y$ plane and the $y-z$ plane, respectively, at a specific time. Tower cranes generally include rope, trolley, tower arms, tower bodies, and base. The model uses the tower body, the tower arm, and the trolley as rigid bodies to explore the vibration characteristics of the swing angle of the payload.

The angle $\theta$ rotates around the $z$-axis, and the angle $\beta$ rotates around the $x$-axis. $L_{y}$ represents the rope length from the trolley to the object. The line length changes continuously during the motion. Moreover, the line length changes during acceleration, constant speed, and deceleration are different. The experimental process set a certain initial angle to validate the simulation. The experiment ignores the influence of wind speed on the swing angle because the experiment is indoors.

Furthermore, the sign $J_{1}$ is the crane payload moment of inertia around the $z$-axis in Figure 1 . The sign $J_{2}$ is the moment of inertia around the $x$-axis. The symbols $e, w$, and $m$, respectively, represent the acceleration of the lifting motor, the acceleration of the luffing motor, and the mass of the payload. The derivation of the equation of motion uses the D'Alembert principle in the following form.

$$
\sum \vec{M}_{O}\left(\vec{F}_{i}\right)+\sum \vec{M}_{O}\left(\vec{F}_{N i}\right)+\sum \vec{M}_{O}\left(\vec{F}_{I i}\right)=0
$$

Here, $\vec{F}_{I i}$ represents the inertial force of $i$ th particle, which is a virtual force. $\vec{F}_{i}$ represents the constraining force 


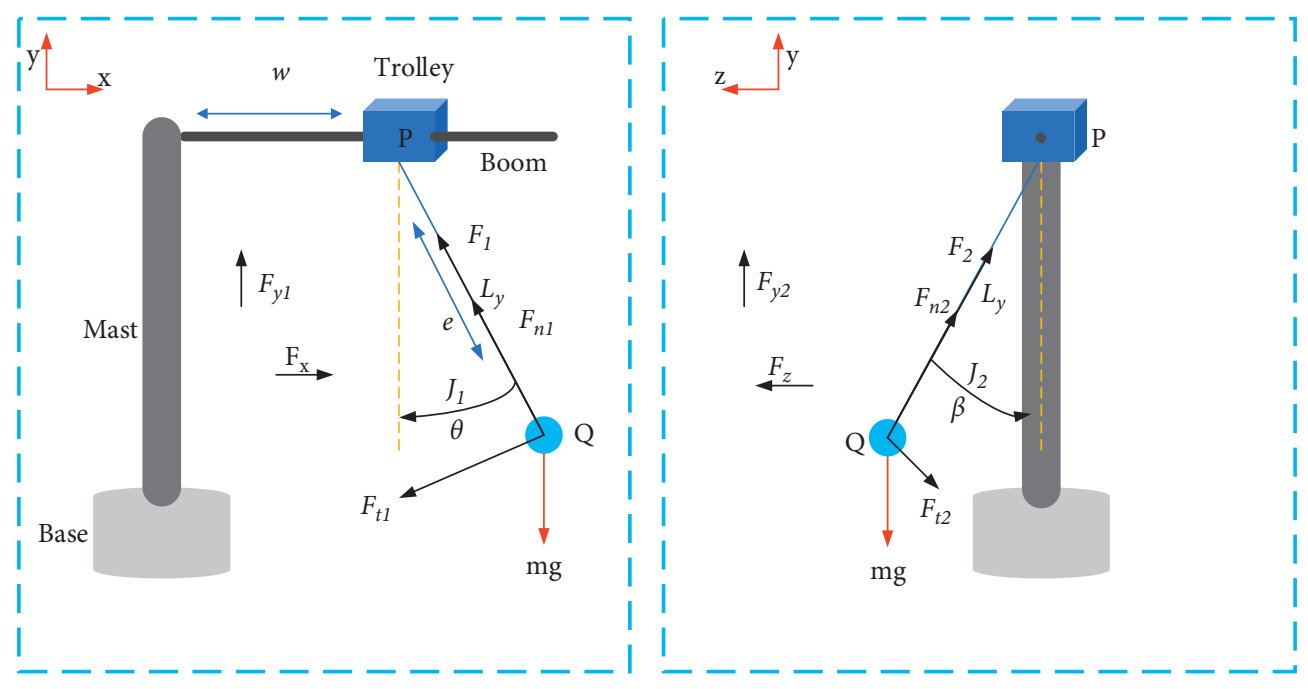

FIgURE 1: Force analysis of LLCM.

of $i$ th particle. $w$ represents the active force of $i$ th particle. The forces of the crane payload are as follows:

$$
\begin{aligned}
\vec{F}_{2} & =m g, \\
\vec{F}_{I 1} & =m L_{y} \ddot{\theta}, \\
\vec{F}_{I 2} & =m L_{y} \ddot{\beta}, \\
\vec{F}_{I 3} & =m L_{y} \dot{\theta}, \\
\vec{F}_{I 4} & =m L_{y} \ddot{\beta}, \\
\vec{F}_{N 1} & =w m, \\
\vec{F}_{N 2} & =m e, \\
\vec{M}_{01} & =J_{1} \ddot{\theta}, \\
\vec{M}_{\mathrm{O} 2} & =J_{2} \ddot{\beta} .
\end{aligned}
$$

The force of the system around point $\mathrm{P}$ has three parts: the active force, the inertial force, and the constraining force. The crane payload is in an equilibrium state of rotating around the point $\mathrm{P}$.

$$
\begin{aligned}
& \vec{F}_{1}-\vec{F}_{N 2}-\vec{F}_{2}(\cos \theta+\cos \beta)-\vec{F}_{N 1} \sin \theta-\vec{F}_{I 3}-\vec{F}_{I 4}=0, \\
F_{x}= & \vec{F}_{2} \sin \theta \cos \beta-\vec{F}_{N 1}+\vec{F}_{N 2} \sin \theta-\vec{F}_{I 1} \cos \theta \\
F_{y}= & -2 \vec{F}_{2} \cos \theta \cos \beta-\vec{F}_{N 2}(\cos \theta+\cos \beta)-\vec{F}_{I 1} \sin \theta-\vec{F}_{I 2} \sin \beta, \\
F_{z}= & \vec{F}_{2} \cos \theta \sin \beta+\vec{F}_{N 2} \sin \beta-\vec{F}_{I 2} \cos \beta
\end{aligned}
$$

$\vec{F}_{1}$ represents the pulling force of the object to the tower arm. The moments of inertia $J_{1}$ and $J_{2}$ are rotating with angular speeds $\dot{\theta}$ and $\dot{\beta}$. When the axis of rotation passes over the end of the rod and is perpendicular to the rod, the moments of inertia equation is as follows:

$$
J=\frac{m L^{2}}{3} .
$$

The symbol $m$ represents the mass of the rod. The symbol $L$ represents the rod length. Point $P$ in Figure 1 is the center of rotation. The crane payload is considered as the length of the section at the end of a thin rod, whose length is $b_{i}$. The equation for the moments of inertia of the crane payload is as follows:

$$
J_{i}=\frac{m\left[L_{y i}^{2}-\left(L_{y i}-b_{i}\right)^{2}\right]}{3} .
$$




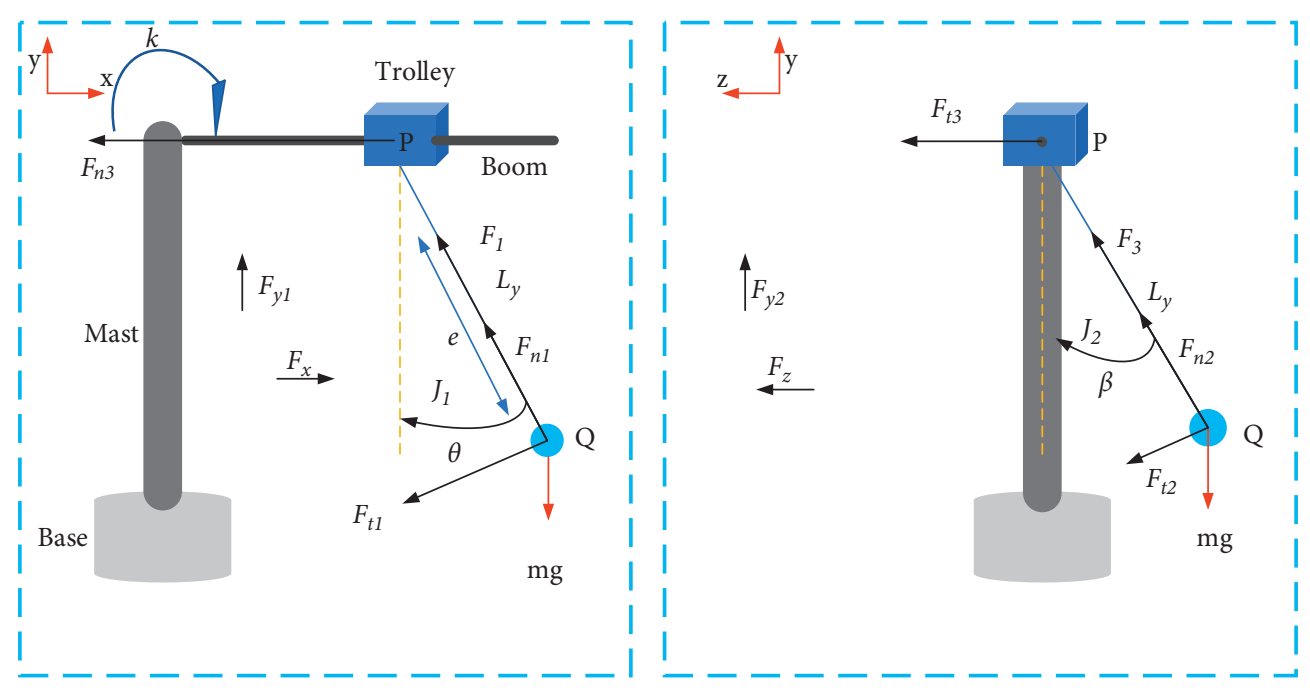

FIGURE 2: Force analysis of LSCM.

2.2. Lifting-Slewing Motion Model. Figure 2 shows a schematic diagram of the lifting and slewing working conditions. An orthogonal coordinate system is inside the schematic diagram. The space model of the lifting-slewing condition is projected to the $x-y$ plane and the $y$ - $z$ plane, respectively, at a specific time. To study the vibration characteristics of the swing angle of payload, tower cranes generally include rope, trolley, tower arms, tower bodies, and base. Tower body, tower arm, and trolley are regarded as rigid bodies.

The angle $\theta$ rotates around the $z$-axis, and the angle $\beta$ rotates around the $x$-axis. $L_{y}$ represents the rope length from the trolley to the object. The line length changes continuously during the motion. Moreover, the line length changes during acceleration, constant speed, and deceleration are different. The experimental process set a certain initial angle to validate the simulation. The experiment ignores the influence of wind speed on the swing angle because the experiment is indoors. $J_{3}$ represents the moment of inertia of the crane payload around the $z$-axis. Also, $J_{4}$ is the moment of inertia of the crane payload around the $x$-axis.

Furthermore, the symbols $a, e$, and $m$ represent the acceleration of the lifting motor, the acceleration of the luffing motor, and the object's mass, respectively.
The derivation of the equation of motion uses the D'Alembert principle in the following form. As is shown in Figure 3, the space model of the lifting-slewing condition is projected to the $x-z$ plane. Point $O$ represents the tower mast. Point $\mathrm{Q}$ represents the crane payload. The $R_{0}$ represents the distance from point $O$ to point $P$. The crane payload has Coriolis acceleration. The forces of crane payload are as follows:

$$
\begin{aligned}
\vec{F}_{I 5} & =m(\dot{k})^{2} R_{1}, \\
\vec{F}_{I 6} & =2 m \dot{k} \dot{\theta} L_{y} \cos \theta, \\
\vec{F}_{N 3} & =m \ddot{k} R_{1} .
\end{aligned}
$$

The symbol $k$ denotes the rotational angle of the tower crane arm. $R_{0}$ represents the distance from the tower to the trolley $R_{1}=R_{0}+L_{y} \sin \theta$. The symbol $\vec{F}_{3}$ represents the pulling force of the payload to the tower arm. The total force around point $\mathrm{P}$ has three components. They are the active force, inertial force, and constraining force. The corresponding equations are as follows:

$$
\begin{aligned}
F_{x}= & \vec{F}_{2} \sin \theta \cos \beta-\vec{F}_{I 5}+\vec{F}_{N 2} \sin \theta-\vec{F}_{I 1} \cos \theta, \\
F_{y}= & -2 \vec{F}_{2} \cos \theta \cos \beta-\vec{F}_{N 2}(\cos \theta+\cos \beta)-\vec{F}_{I 1} \sin \theta-\vec{F}_{I 2} \sin \beta, \\
F_{z}= & -\vec{F}_{2} \cos \theta \sin \beta-\vec{F}_{N 2} \sin \beta+\vec{F}_{N 3}-\vec{F}_{I 2} \cos \beta+\vec{F}_{I 6}, \\
& \vec{F}_{3}-\vec{F}_{N 2}-\vec{F}_{2}(\cos \theta+\cos \beta)-\vec{F}_{I 3}-\vec{F}_{I 4}+\vec{F}_{I 5} \sin \theta-\vec{F}_{N 3} \sin \beta-\vec{F}_{I 6} \sin \beta=0 .
\end{aligned}
$$

After substituting corresponding expressions into equation (1), the matrix below shows the derivation result for the differential equations of motion of the mathematical model.

$$
\left[\begin{array}{ll}
A_{11} & A_{12} \\
A_{21} & A_{22} \\
A_{31} & A_{32} \\
A_{41} & A_{42}
\end{array}\right]\left[\begin{array}{l}
\ddot{\theta} \\
\ddot{\beta}
\end{array}\right]=\left[\begin{array}{l}
B_{1} \\
B_{2} \\
B_{3} \\
B_{4}
\end{array}\right] .
$$




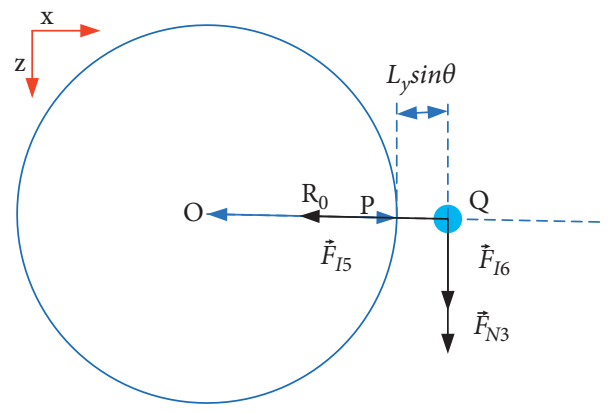

FIGURE 3: Force analysis of LSCM on the $x-z$ plane.
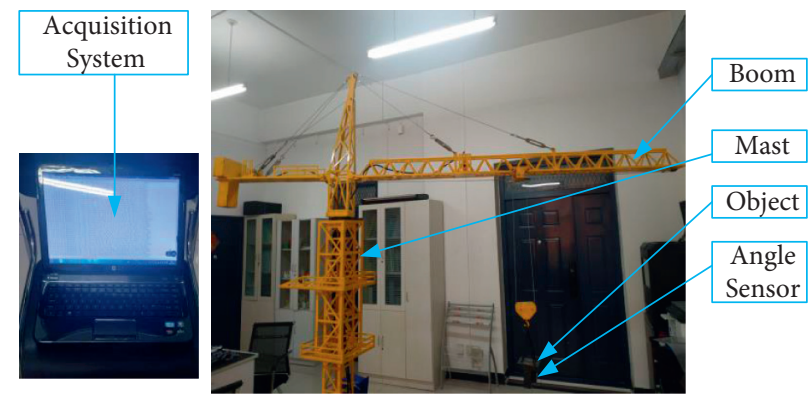

FIgURE 4: Components of the test bench.
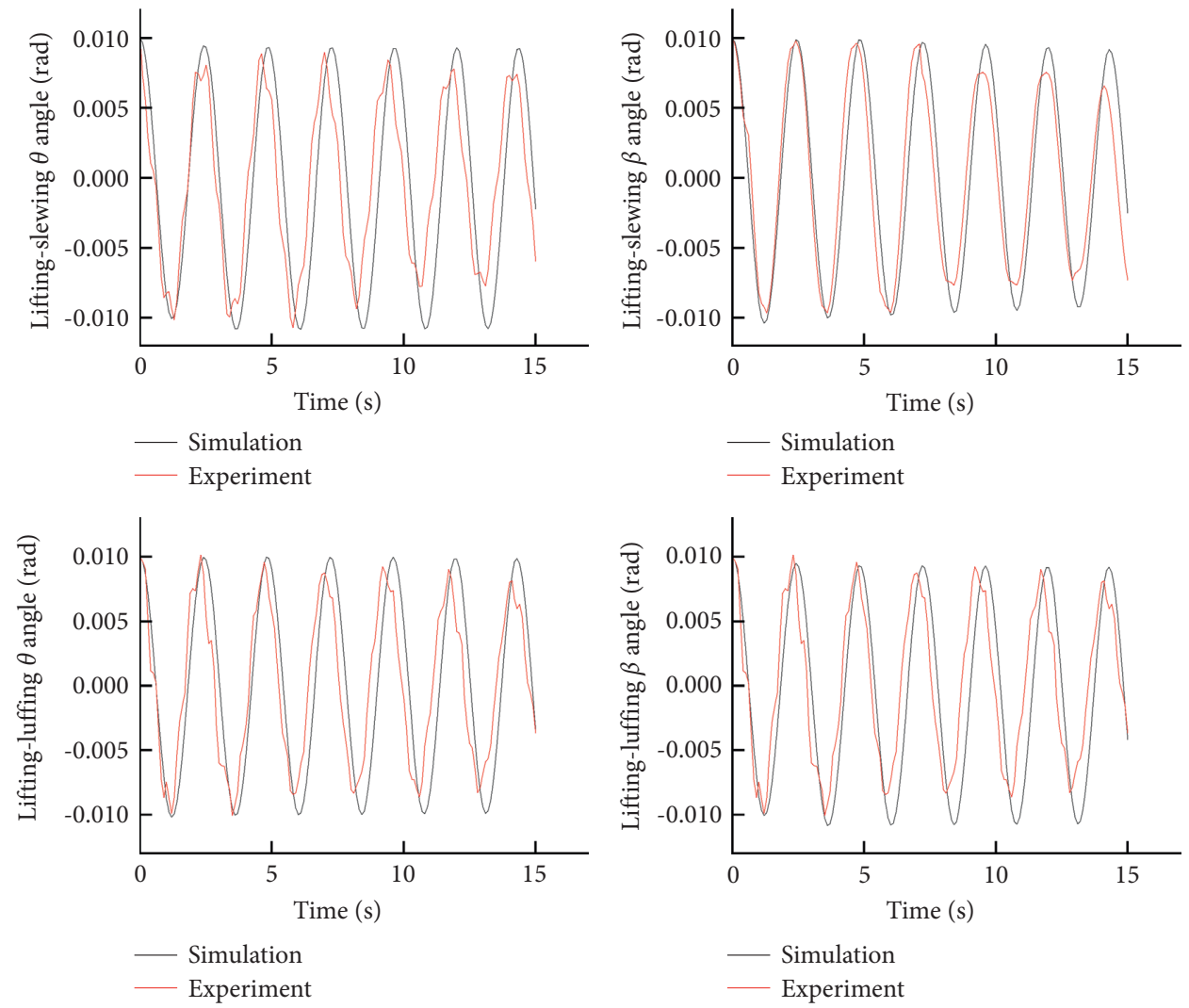

- Simulation

FIGURE 5: Experimental and simulated swing angles. 
TABle 1: Time-domain indicators.

\begin{tabular}{lr}
\hline Abbreviation & Mathematical expression \\
RMS & $X_{\mathrm{rms}}=\sqrt{(1 / N) \sum_{i=1}^{N} x_{i}^{2}}$ \\
PV & $X_{\mathrm{pv}}=\left(N \max \left|x_{i}\right|\right) /\left(\sum_{i=1}^{N}\left|x_{i}\right|\right)$ \\
KV & $X_{\mathrm{kv}}=(1 / N) \sum_{i=1}^{N}\left(\left(x_{i}-\bar{x}\right) / \sigma\right)^{4}$ \\
SV & $X_{s v}=(1 / N) \sum_{i=1}^{N}\left(\left(x_{i}-\bar{x}\right) / \sigma\right)^{3}$ \\
\hline
\end{tabular}

TABle 2: Frequency-domain indicators.

\begin{tabular}{lr}
\hline Abbreviation & Mathematical expression \\
\hline FC & $X_{\mathrm{fc}}=(1 / N) \sum_{i=1}^{N} f_{i}$ \\
RVF & $X_{\mathrm{rvf}}=\sqrt{(1 / N) \sum_{i=1}^{N}\left(f_{i}-x_{f c}\right)^{2}}$ \\
RMSF & $X_{\mathrm{rmsf}}=\sqrt{(1 / N) \sum_{i=1}^{N} f_{i}^{2}}$ \\
\hline
\end{tabular}
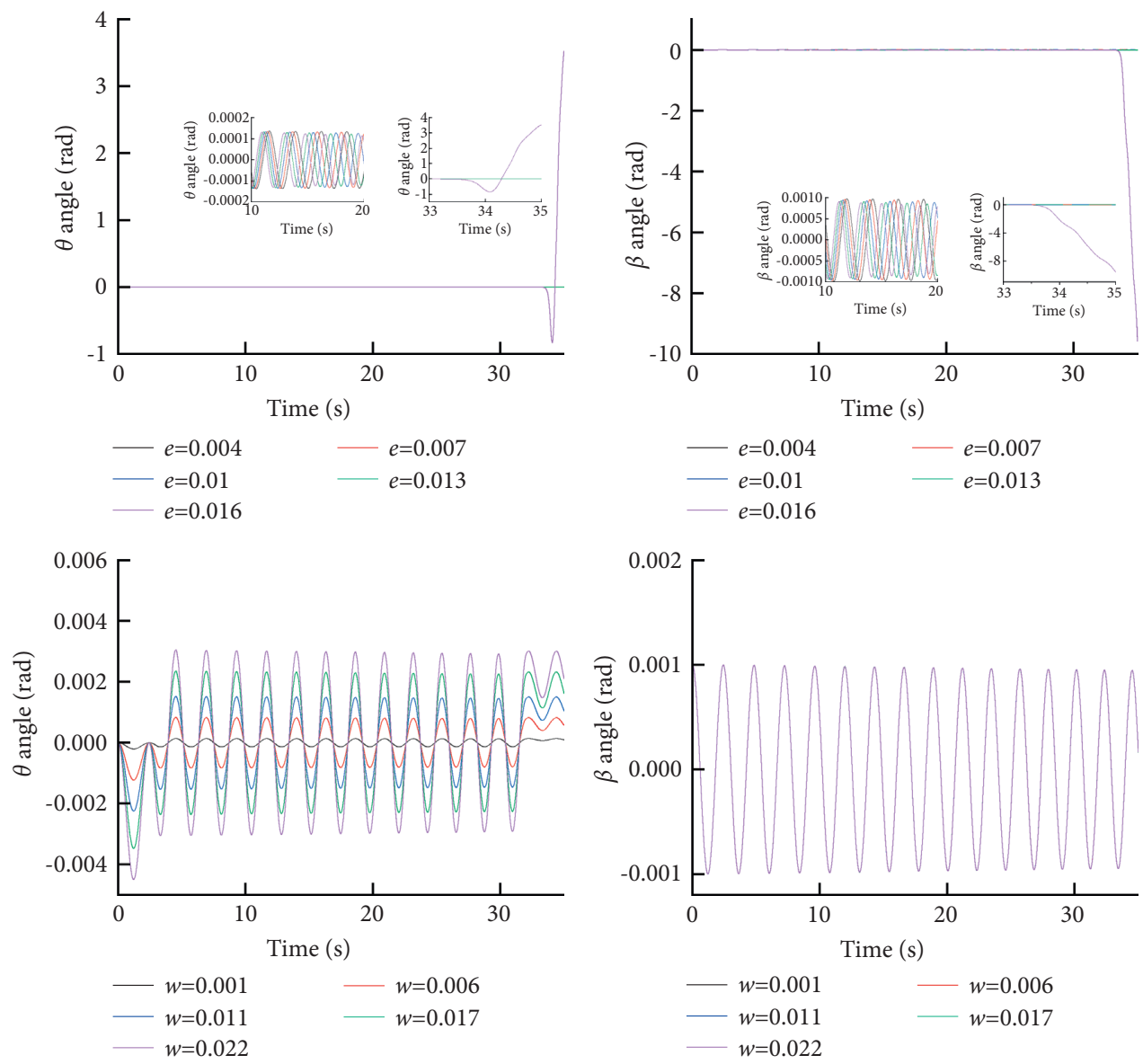

Figure 6: Response of $e$ and $w$ to the LLCM.

The elements $A_{i j}$ and $B_{i}$ are defined in Appendix. Equation (8) represents four second-order nonlinear differential equations with nonconstant coefficients. The elements $B_{1}$ and $B_{2}$ are in the LLCM. The elements $B_{3}$ and $B_{4}$ are in the LSCM.
2.3. Model Verification. It is not easy to test, verify, and collect data due to the large size of the tower crane. Therefore, the laboratory uses a scaled-down tower crane. The design needs to follow the requirements of structure and materials in Figure 4. The validation of the LSCM and LLCM 

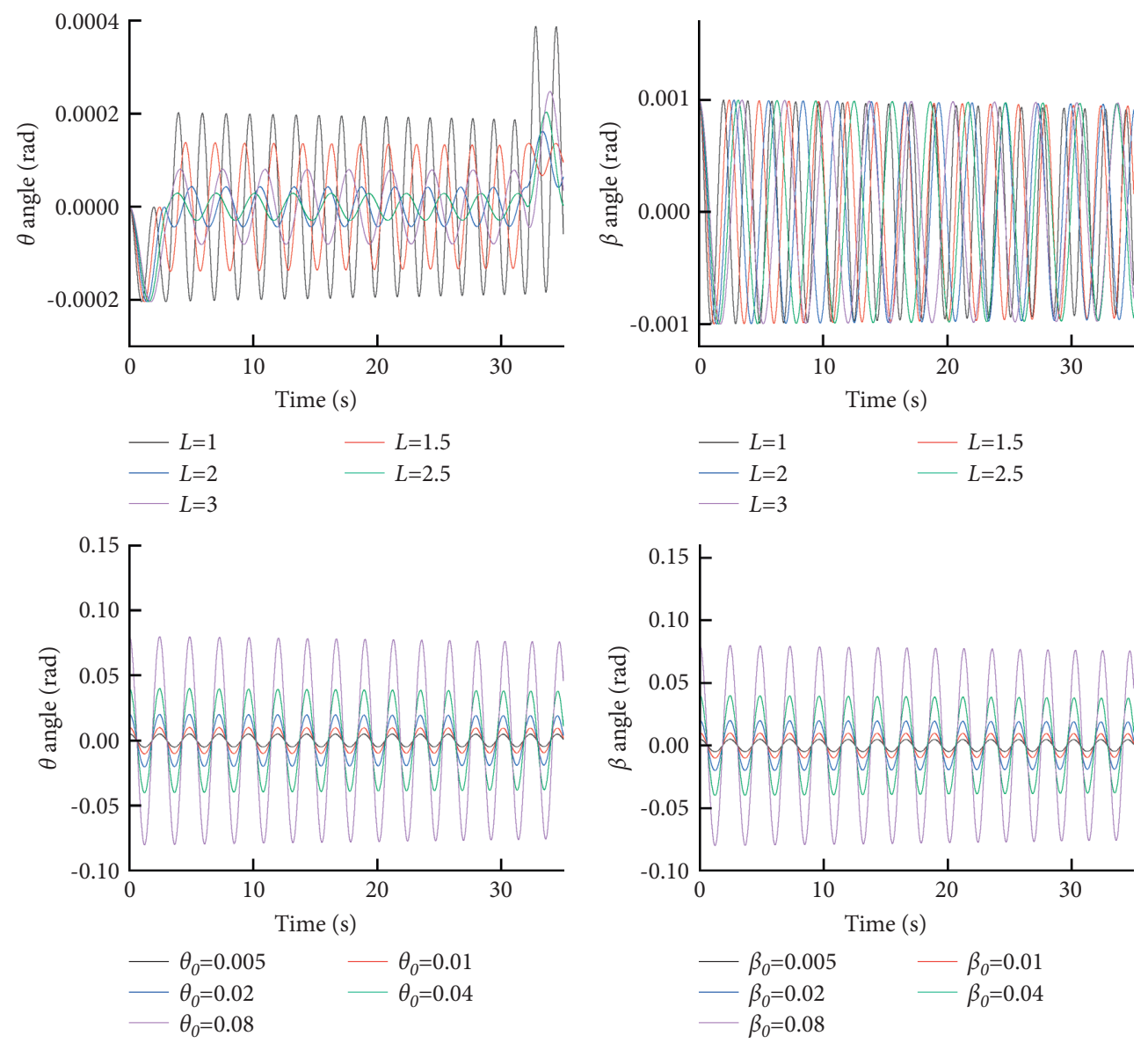

Figure 7: Response of $L, \theta_{0}$, and $\beta_{0}$ to the LLCM.

models applies the comparison between the experiment and simulation results. Both the experiment and the simulation process experience from accelerating to a constant speed and then decelerating. The mass is $1.45 \mathrm{~kg}, 2.4 \mathrm{~kg}$, and $6.6 \mathrm{~kg}$, respectively, in the experiment. The lifting, luffing, and slewing acceleration are $0.003 \mathrm{~m} / \mathrm{s}^{2}, 0.001 \mathrm{~m} / \mathrm{s}^{2}$, and $0.02 \mathrm{~m} /$ $\mathrm{s}^{2}$, respectively. The rope length is $1.5 \mathrm{~m}$. The lifting-luffing motion and the lifting-slewing motion are the two primary components of the experiment. Figure 5 shows the results of the two movement states. The experiment and simulation under the two working conditions start from the maximum value of swing angle $\theta$ and $\beta$. The simulation data are slightly larger than the experiment when they decelerate from the 10 th second, which may be due to other components' vibration. In the lifting and luffing conditions, when the initial angle of the angles $\theta$ and $\beta$ is $0.01 \mathrm{rad}$, the swing variation of the angles $\theta$ and $\beta$ is similar. Thus, on the whole, the nonlinear simulation is a good predictor of crane performance.

\section{Motion Response and Analysis}

3.1. Indicator Analysis. This paper uses four time-domain indicators to analyze the influence of parameter changes on the spatial angle signal waveform. They are the root mean square value (RMS), peak value (PV), kurtosis value (KV), and skewness value (SV). In addition, there are also three frequency-domain indicators. They are the frequency center (FC), root mean square frequency (RMSF), and frequency standard deviation (RVF) [31]. Table 1 shows the timedomain indicators. Table 2 shows the frequency-domain indicators.

3.2. Lifting-Luffing Motion Response. As shown in Figure 6, the lifting acceleration $e$ is, respectively, taken as $0.004 \mathrm{~m} / \mathrm{s}^{2}$, $0.007 \mathrm{~m} / \mathrm{s}^{2}, 0.01 \mathrm{~m} / \mathrm{s}^{2}, 0.013 \mathrm{~m} / \mathrm{s}^{2}$, and $0.016 \mathrm{~m} / \mathrm{s}^{2}$. The luffing acceleration $w$ is $0.001 \mathrm{~m} / \mathrm{s}^{2}, 0.006 \mathrm{~m} / \mathrm{s}^{2}, 0.011 \mathrm{~m} / \mathrm{s}^{2}, 0.017 \mathrm{~m} /$ $\mathrm{s}^{2}$, and $0.022 \mathrm{~m} / \mathrm{s}^{2}$, respectively, in the LLCM. The dynamic response of the angles $\theta$ and $\beta$ can have a divergence phenomenon when the lifting acceleration is $0.016 \mathrm{~m} / \mathrm{s}^{2}$. Also, the significant acceleration leads to a rapid increase of the swing angle, which seriously reduces the stability of the tower crane. The angle $\theta$ rises with the increase of the luffing acceleration, and the angle $\beta$ does not change significantly. Maximum angular responses are during acceleration with varying luffing acceleration. The angle response cycles are constantly changing with varying lifting acceleration $e$. However, the angle response cycles are constant with varying acceleration $w$.

In Figure 7, the rope length $L$ is $1 \mathrm{~m}, 1.5 \mathrm{~m}, 2 \mathrm{~m}, 2.5 \mathrm{~m}$, and $3 \mathrm{~m}$, respectively, under the lifting-luffing condition. The initial angle is, respectively, taken as $0.005 \mathrm{rad}, 0.01 \mathrm{rad}$, 

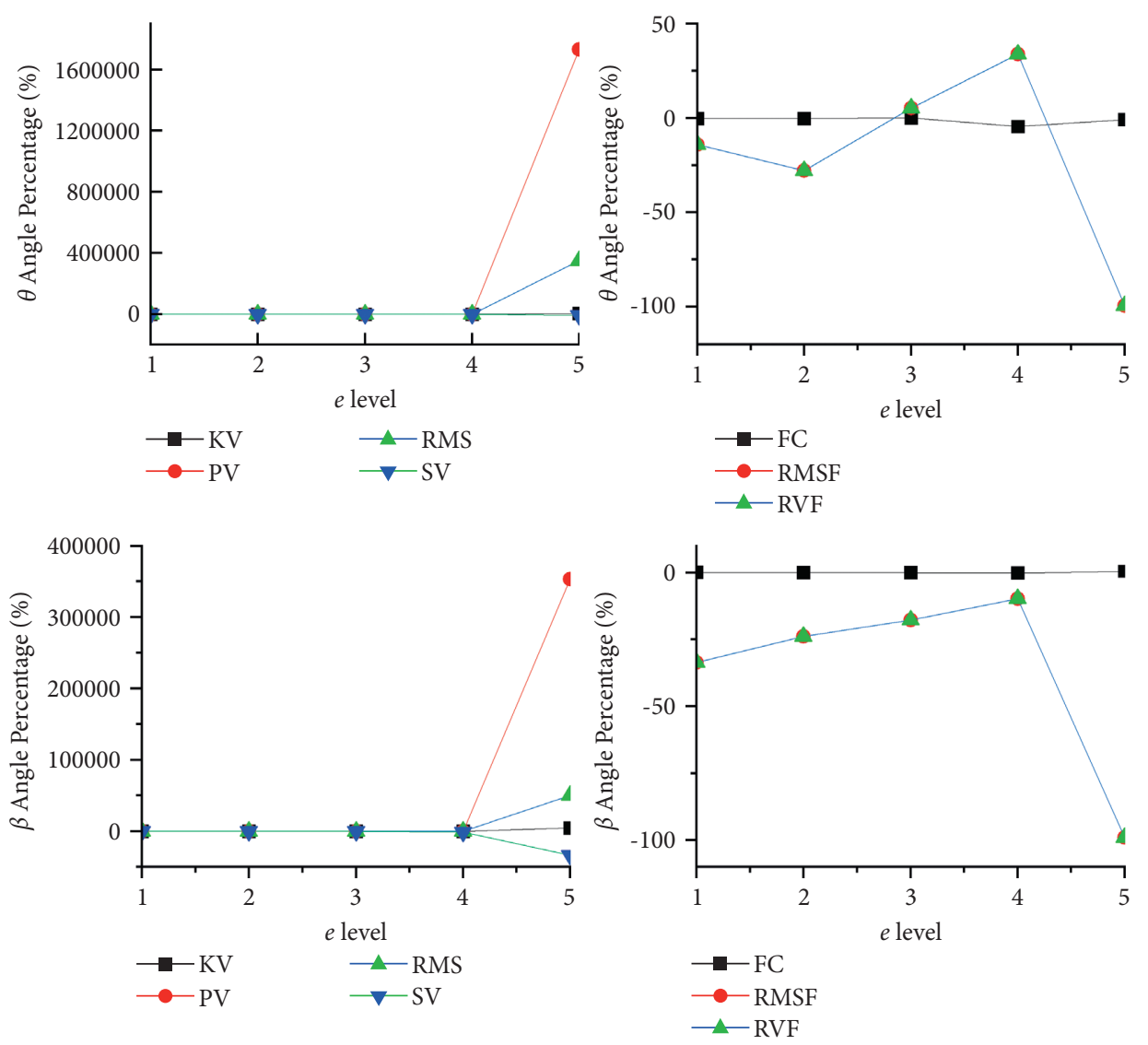

FIGURE 8: Effect of the lifting acceleration on the lift-luffing angles.

$0.02 \mathrm{rad}, 0.04 \mathrm{rad}$, and $0.08 \mathrm{rad}$. As the rope length changes, the dynamic response of the angle $\theta$ fluctuates greatly during the deceleration phase. The swing angle $\theta$ gradually decreases with the increase of the rope length. The swing angle $\theta$ rises among the positive half axis when the rope length $L$ is $1 \mathrm{~m}$. The angle $\theta$ tends to diverge with the $3 \mathrm{~m}$ rope length. The angle $\beta$ response does not change much. The angles $\theta$ and $\beta$ response cycle keep changing with adjusting the rope length. With the increase of the initial angles, the angles $\theta$ and $\beta$ response also have an upward trend. The maximum angle is the initial angle during the response. The angle response cycles are constant with varying initial angles.

3.3. Lifting-Luffing Motion Analysis. Figure 8 shows that the $\mathrm{KV}$ of angle is stable with the change of the lifting acceleration $e$. However, the RMSF and RVF of the angle is sensitive to changes. The PV and RMS can characterize the state change of the angle $\theta$ well in the later stage, but their stability is worse than the RMS. The FC of the angle $\theta$ is stable. The RMSF and RVF experience decrease, increase, and decrease successively. The changes of the RMS and PV of the angle $\beta$ are the same as that of the angle $\theta$. The KV has similar changes. The KV, RMS, and PV appear in the later stage. The FC of the angle $\beta$ has good stability. The RMSF and RVF increase in the early stage and hugely decrease in the later stage. The above analysis shows that when PV level 5 increases by tens of thousands of times, PV can reflect the phenomenon of angle divergence. RMSF and RVF have dropped by $100 \%$, showing that the acceleration has divergence at level 5 . The lift-luffing angle is stable when the lifting acceleration is in the range from $0.004 \mathrm{~m} / \mathrm{s}^{2}$ to $0.01 \mathrm{~m} /$ $\mathrm{s}^{2}$. FC can monitor the parameter change. PV from level 4 to level 5 increases by tens of thousands of times; PV can reflect the phenomenon of large angle growth. PV, RMS, FC, and RMSF are more sensitive among them.

Figure 9 indicates the effect of the parameter $w$ variation. $\mathrm{KV}$ of the angle $\theta$ is stable. The PV and RMS in the early stage are more stable than in the later stage. The PV and RMS change in the later stage, which can better characterize the changes in response. The FC of the angle $\theta$ is stable. The RMSF and RV decrease successively. They can better characterize the changes in response. Furthermore, the four indicators of the angle $\beta$ time-domain increase in the later stage. The three indicators of the angle $\beta$ frequency-domain are stable. The RMSF and RVF decrease first and then increase. And their change is small. According to the analysis above, the lift-luffing angle is stable when the luffing acceleration is in the range from $0.001 \mathrm{~m} / \mathrm{s}^{2}$ to $0.017 \mathrm{~m} / \mathrm{s}^{2}$. $\mathrm{PV}$, RMS, FC, and RMSF are more sensitive among them.

As shown in Figure 10, when the rope length $L$ is changed, KV and SV first increase and then decrease. RMS and PV first decrease and then increase. The SV can 

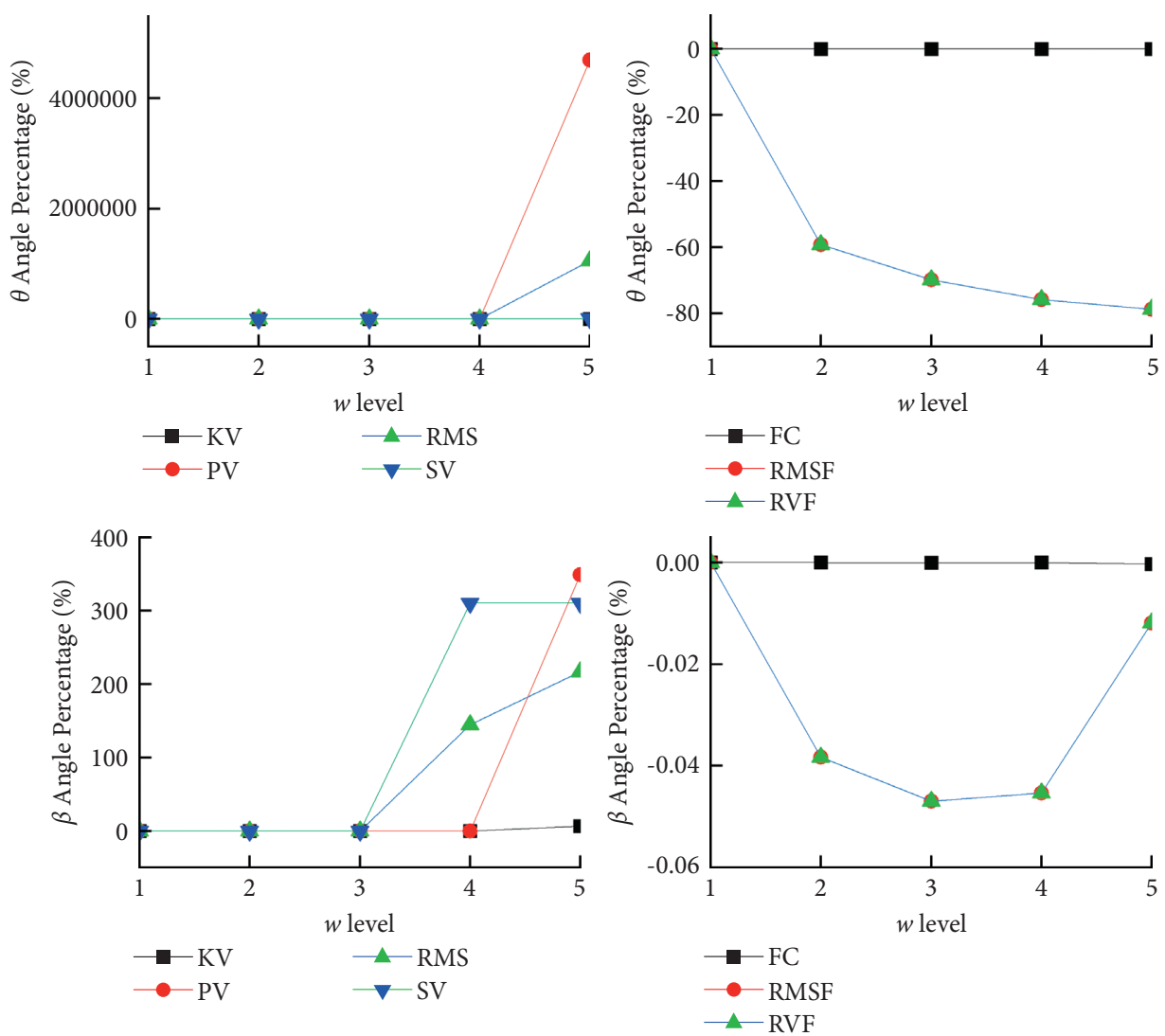

Figure 9: Effect of the luffing acceleration on the lift-luffing angles.
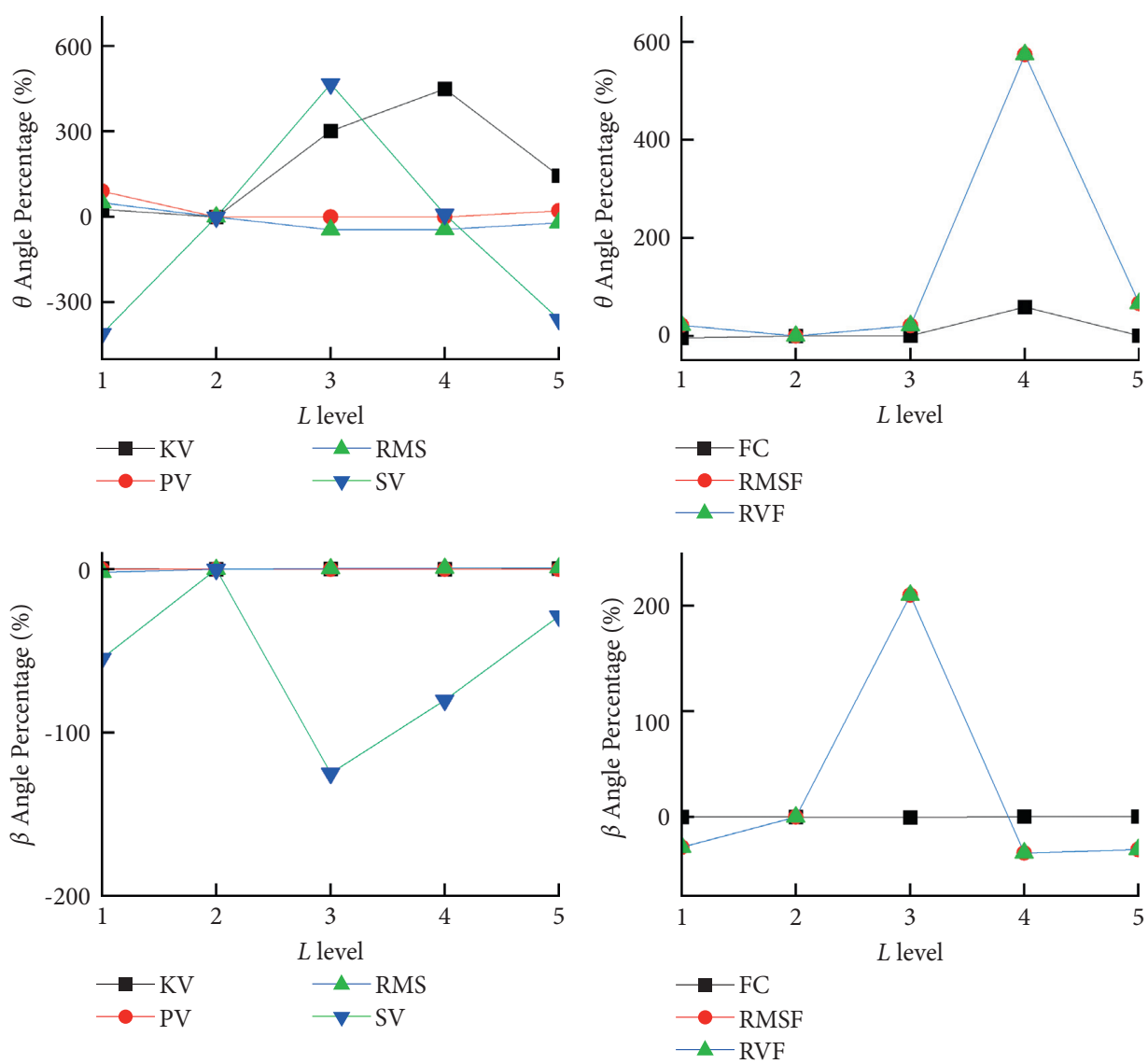

Figure 10: Effect of the rope length on the lift-luffing angle. 

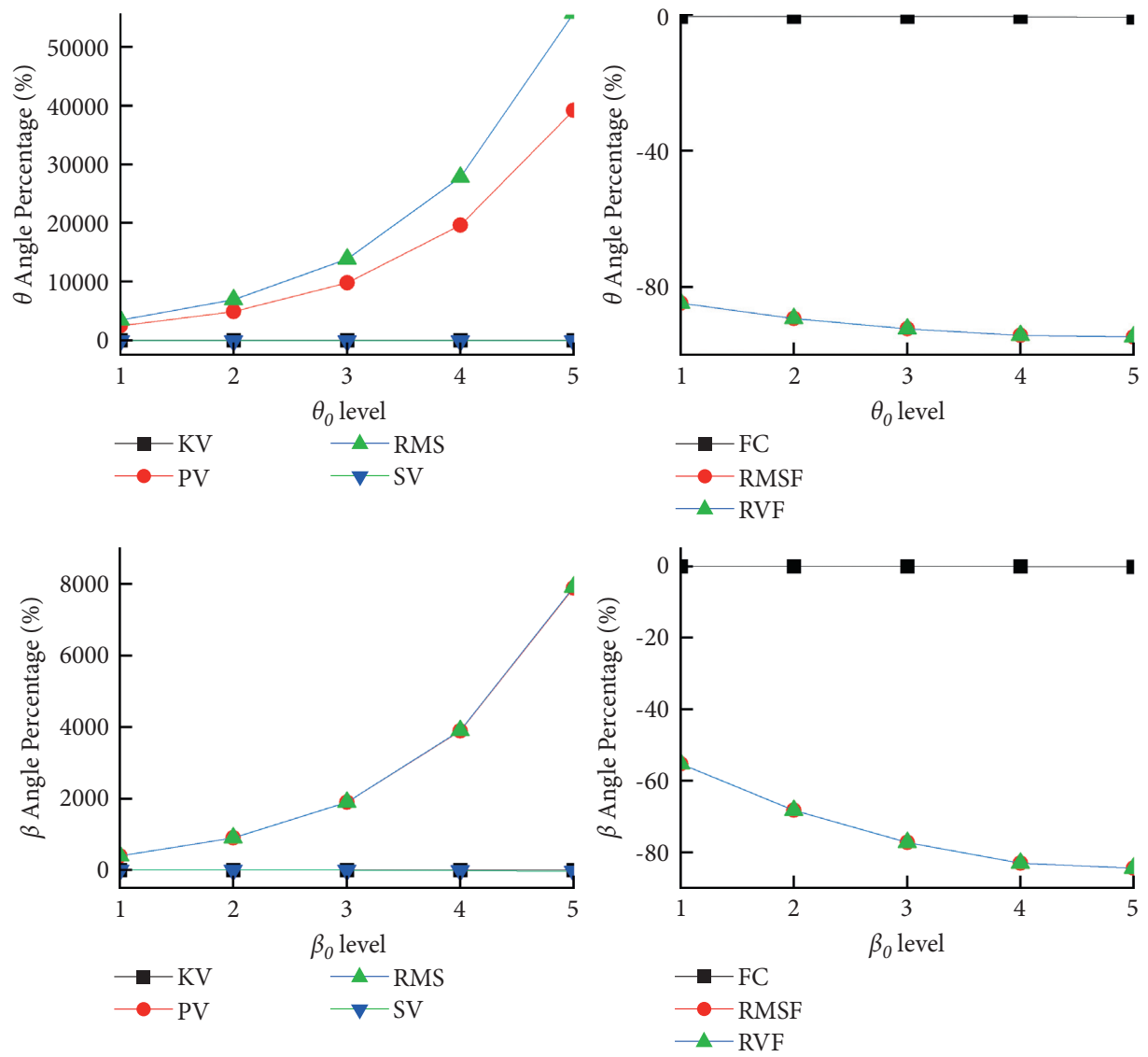

Figure 11: Effect of the initial angles on the lift-luffing angle.

characterize changes in response. The RMSF and RVF of the angle $\theta$ first decrease, then increase, and finally decrease. For the four indicators of the angle $\beta$ time-domain, SV presents the best sensitivity while the PV, KV, and RMS exhibit nonmonotonic characteristics, which are not suitable for construction safety describing. The RMSF and RVF of the angle $\beta$ first increase, then decrease, and finally increase. The above analysis shows that the higher the tower crane is, the stronger the swing angle fluctuation is for the same lifting height. SV, FC, and RMSF are more sensitive among them.

As shown in Figure 11, the initial angle of the parameter is adjusted. The KV, PV, RMS, and SV of the angle $\theta$ are monotonic. On the other hand, the RMSF and RVF of the angle $\theta$ have been decreasing. The time-domain and frequency-domain indicator changes of the angle $\beta$ are similar to the angle $\theta$. The above analysis indicates that the initial angle change can monitor the angle response change through PV, RMS, RMSF, and RVF.

3.4. Lifting-Slewing Motion Response. In Figure 12, the parameter lifting acceleration $e$ is, respectively, taken as $0.004 \mathrm{~m} / \mathrm{s}^{2}, 0.007 \mathrm{~m} / \mathrm{s}^{2}, 0.01 \mathrm{~m} / \mathrm{s}^{2}, 0.013 \mathrm{~m} / \mathrm{s}^{2}$, and $0.016 \mathrm{~m} / \mathrm{s}^{2}$, and the parameter luffing acceleration $a$ is, respectively, taken as $0.01 \mathrm{~m} / \mathrm{s}^{2}, 0.025 \mathrm{~m} / \mathrm{s}^{2}, 0.04 \mathrm{~m} / \mathrm{s}^{2}, 0.055 \mathrm{~m} / \mathrm{s}^{2}$, and $0.07 \mathrm{~m} / \mathrm{s}^{2}$ in the lifting-slewing condition. By changing the lifting acceleration, the dynamic response of angles $\theta$ and $\beta$ diverges from $0.016 \mathrm{~m} / \mathrm{s}^{2}$, which seriously reduces the stability of the tower crane. The angle response cycles reduce with lifting accelerations increase. When the slewing acceleration is changed, it is obvious that angles $\theta$ and $\beta$ increase as the slewing acceleration increases. The dynamic response of the angle $\beta$ can have a divergence phenomenon when the lifting acceleration starts from $0.04 \mathrm{~m} / \mathrm{s}^{2}$. The angle response cycles are constant with varying slewing accelerations.

In Figure 13, the rope length $L$ takes the values $1 \mathrm{~m}$, $1.5 \mathrm{~m}, 2 \mathrm{~m}, 2.5 \mathrm{~m}$, and $3 \mathrm{~m}$, respectively, under the liftingslewing condition. The initial angle of the parameter is, respectively, $0.005 \mathrm{rad}, 0.01 \mathrm{rad}, 0.02 \mathrm{rad}, 0.04 \mathrm{rad}$, and $0.08 \mathrm{rad}$. When the parameter $L$ is adjusted, there is a decreasing state about the dynamic response of angles $\theta$ and $\beta$ in the LSCM, and the angle $\beta$ tends to increase with the $3 \mathrm{~m}$ rope length. The angle response cycles keep changing by adjusting the rope lengths. The initial angle of the parameter is changed. The angle $\theta$ increases with the increase of the initial angle. Although the angle $\beta$ also increases with the increase of the initial angle, the angle $\beta$ starts to diverge from $0.01 \mathrm{rad}$. The angle response cycles are constant with varying initial angles.

3.5. Lifting-Slewing Motion Analysis. Figure 14 displays the effect of the lifting acceleration variation. The KV and SV of the angle $\theta$ are stable, and the RMS and PV are less stable 

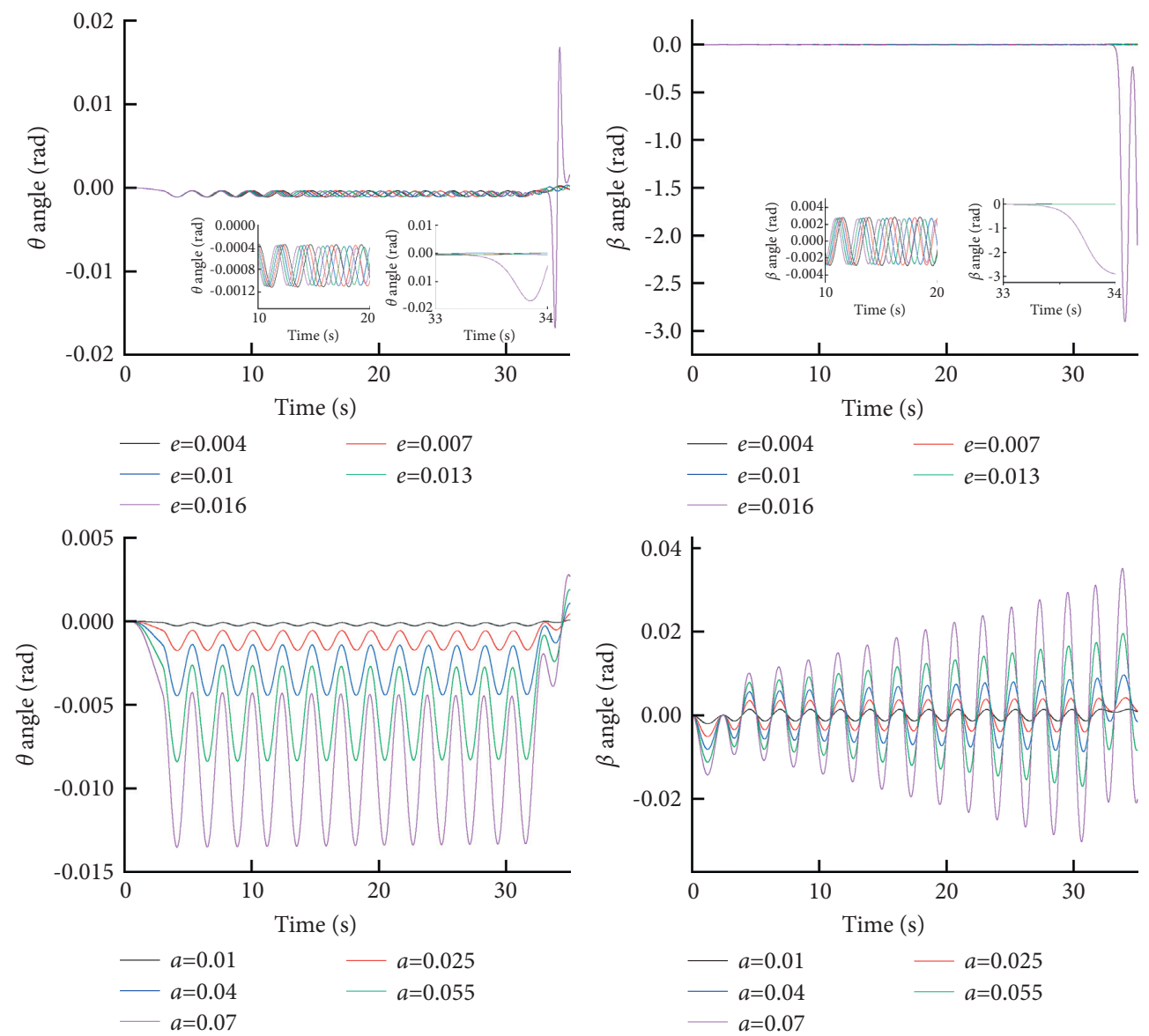

Figure 12: Response of $a$ and $e$ to the LSCM.

than the KV and SV. The RMSF and RVF of the angle $\theta$ show an overall downward trend. Their lifting acceleration level 5 decreases by $-58 \%$ and exhibits the best performance. The $\mathrm{SV}$ of the angle $\beta$ first increases and then decreases. The KV of lifting acceleration level 5 increases by $3422 \%$. The RMSF and RVF first increase, then decrease, and finally decrease. The above analysis shows that the value range of the lifting acceleration is from $0.004 \mathrm{~m} / \mathrm{s}^{2}$ to $0.01 \mathrm{~m} / \mathrm{s}^{2}$, which is relatively stable. The dynamic response of angles $\theta$ and $\beta$ diverges from $0.016 \mathrm{~m} / \mathrm{s}^{2}$. When the PV of lifting acceleration level 5 increases by $7059 \%$ relative to the state, PV can reflect the phenomenon of angle divergence. The lifting acceleration change can monitor the angle response change through $\mathrm{PV}$ of angle $\theta, \mathrm{KV}$ of angle $\beta, \mathrm{RMSF}$, and RVF.

As shown in Figure 15, when the swing acceleration is adjusted, the RMS and PV of the angle $\theta$ increase successively. KV and SV of the angle $\theta$ decrease successively. The FC of the angle $\theta$ frequency is nonmonotonic. The RMSF and RVF decrease successively. The RMS, PV, RMSF, and $\mathrm{RVF}$ are sensitive. The four time-domain indicators of the angle $\beta$ are monotonic. RMS increases, and others decrease. When the RMS of slewing acceleration level increases by $114 \%$, angle $\beta$ has a divergence phenomenon. The RMSF and $\mathrm{RVF}$ of the angle $\beta$ continuously decrease. The above analysis shows that PV, SV, FC, and RMSF are more sensitive than others.

Figure 16 shows the effect of the rope length $L$ on the liftslewing angle. The KV and SV of the angle $\theta$ first decrease, then increase, and then decrease. The KV and SV can characterize the change response of the angle $\theta$. The RMSF and RVF of the angle $\theta$ first increase, then decrease, and finally increase. The SV of the angle $\beta$ first increases, then decreases, and finally increases. The SV fluctuates wildly. The RMSF and RVF of the angle $\beta$ have the same changing trend, from increase to decrease. It can be seen from the above analysis that the rope length $L$ fluctuates sharply between $1.5 \mathrm{~m}$ and $2.5 \mathrm{~m}$.

Figure 17 shows the effect of the initial angle change under the lifting-slewing condition. The KV, PV, RMS, and SV of the angle $\theta$ are monotonic. The KV decreases successively, and others increase successively. They can characterize changes in swing angle response. RMS is the most sensitive among them. The RMSF and RVF of angle $\theta$ are more sensitive than $\mathrm{FC}$. The $\mathrm{KV}$ of angle $\beta$ experiences rise to fall. Others of angle $\beta$ are monotonic. FC slightly increases. RMSF and RVF continuously experience fall. According to the analysis above, PV, RMS, RMSF, and RVF can characterize the angle change. 

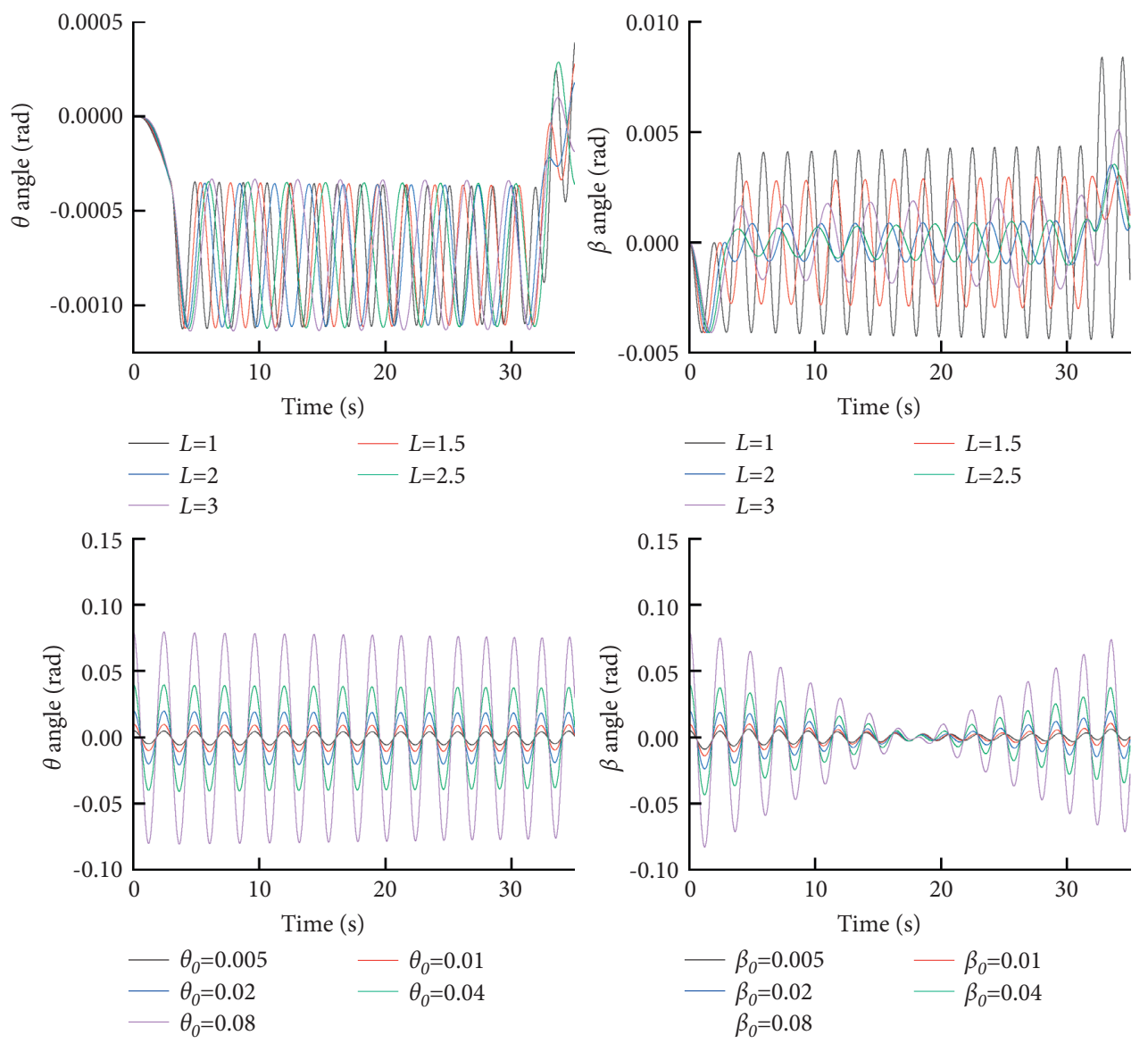

Figure 13: Response of $L, \theta_{0}$, and $\beta_{0}$ to the LSCM.
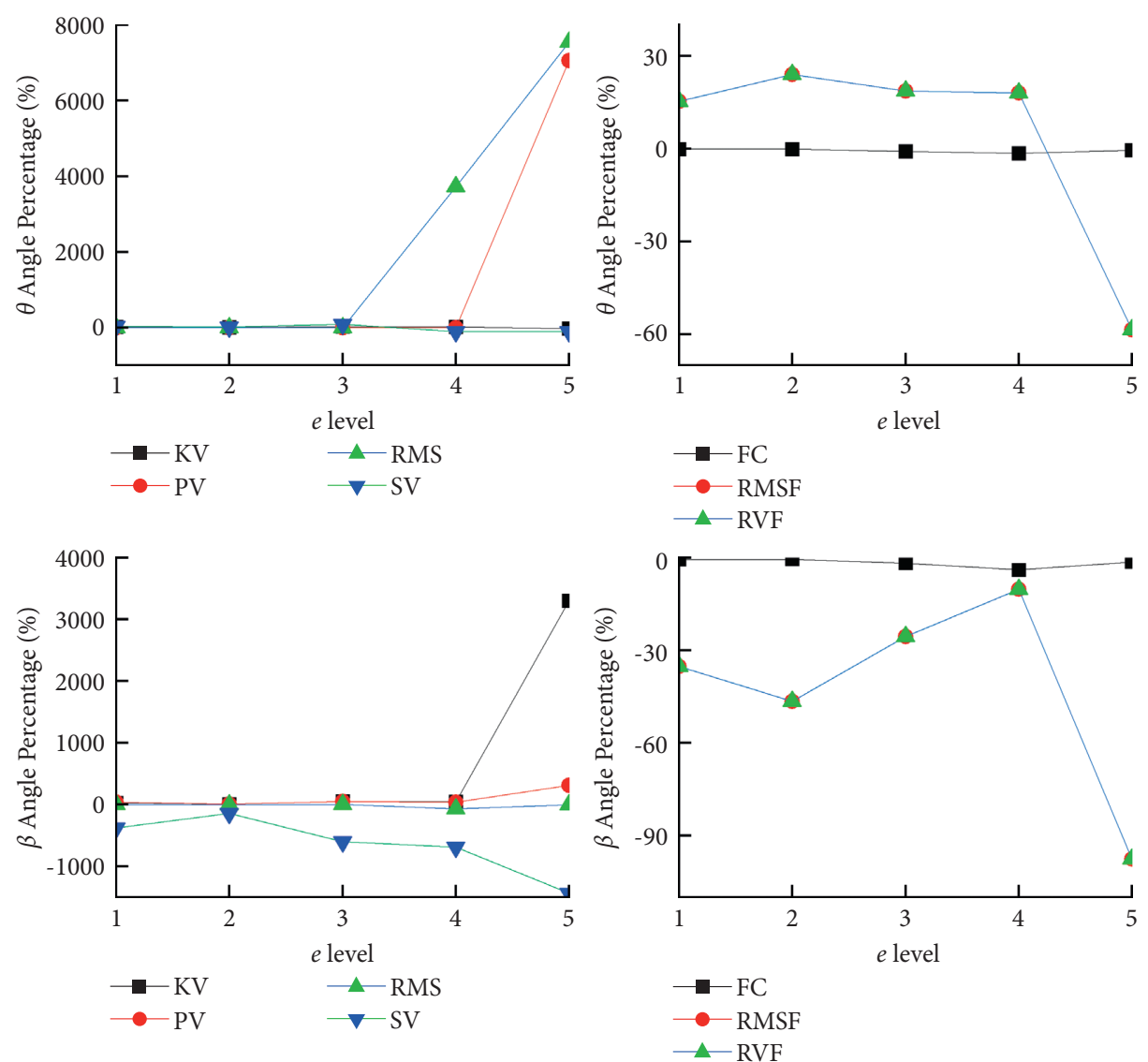

FigURE 14: Effect of the lifting acceleration on the lifting-slewing angle. 

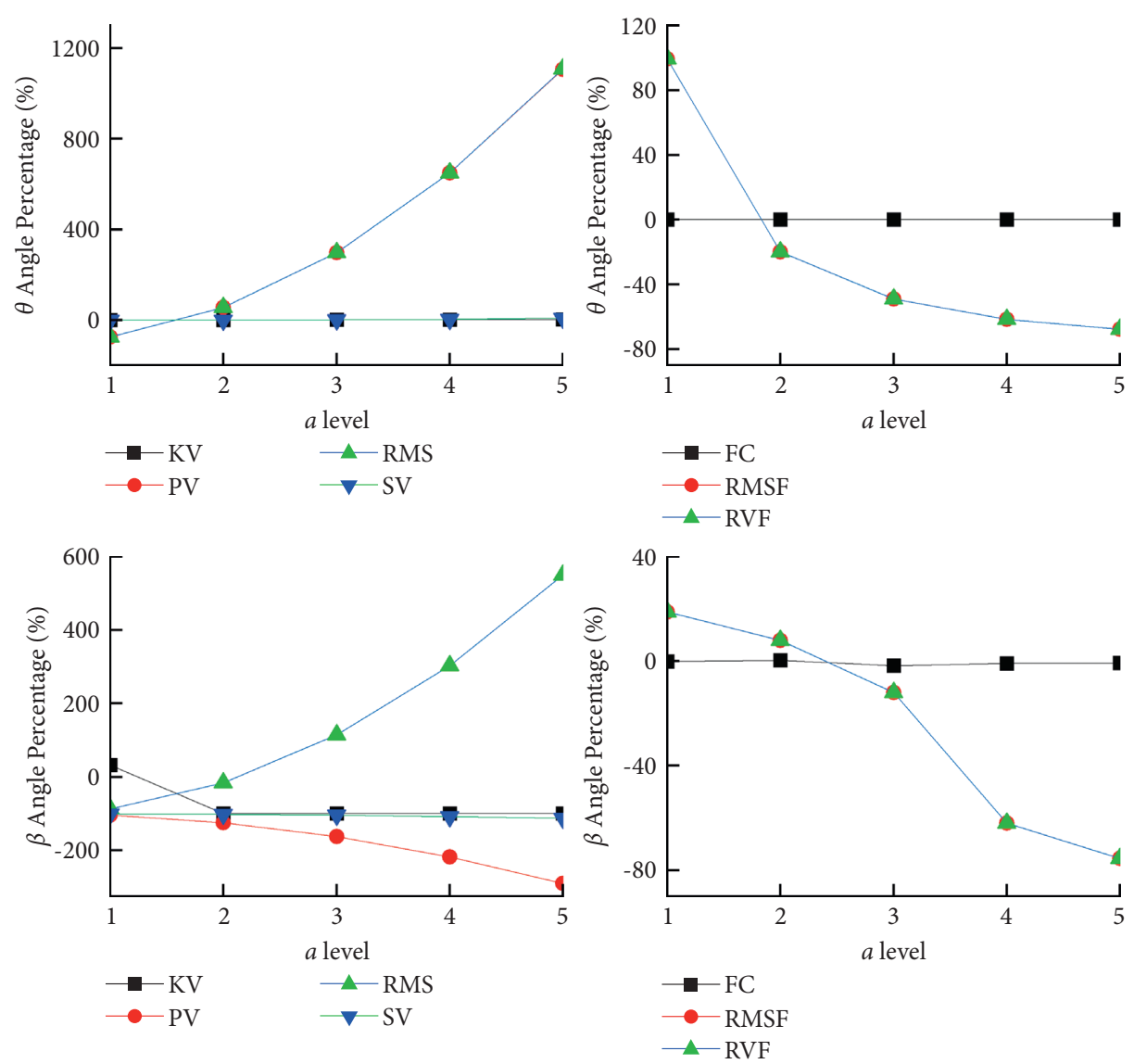

FIGURE 15: Effect of the swing acceleration on the lifting-swing angle.
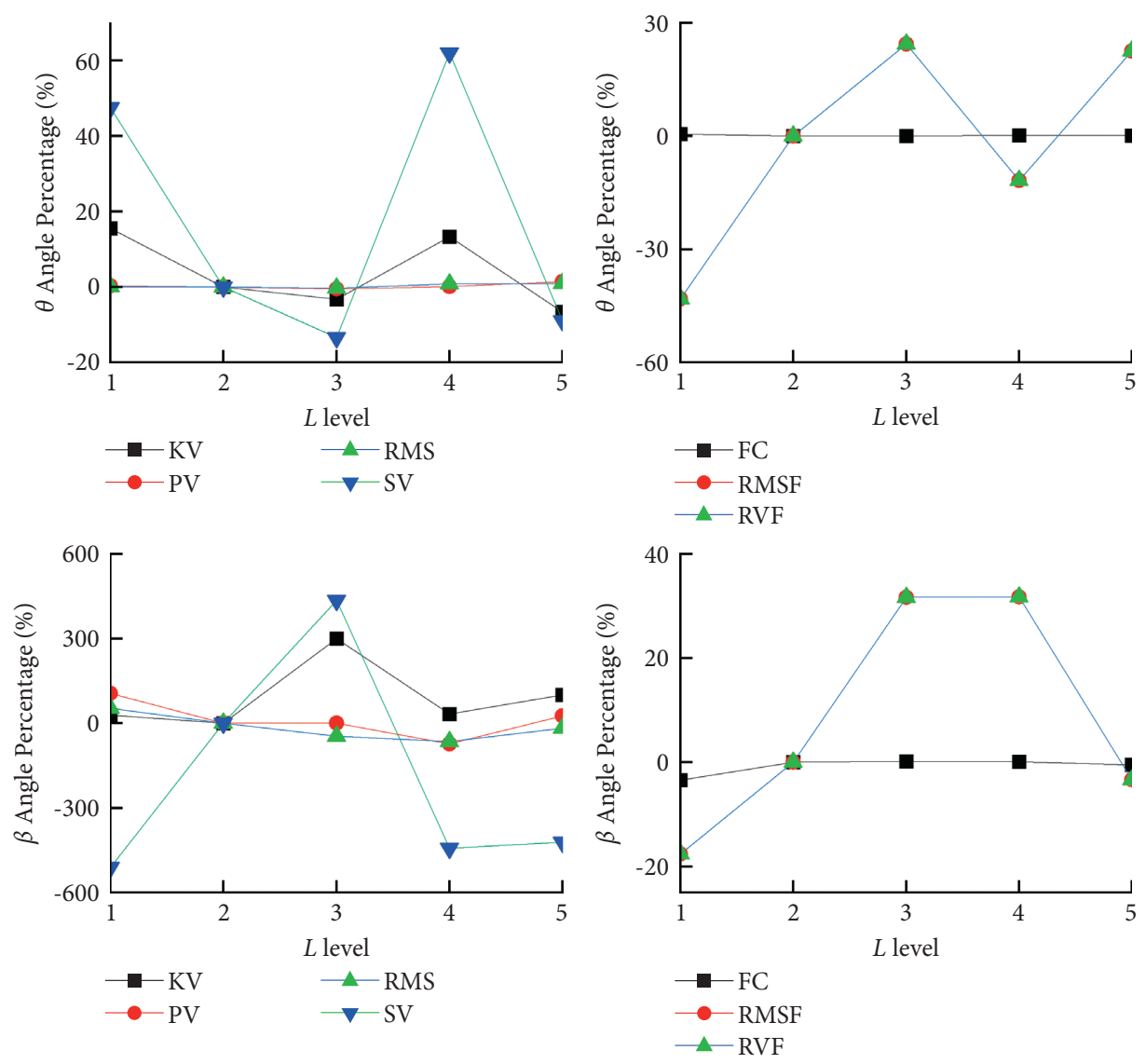

Figure 16: Effect of the rope length on the lift-slewing angle. 

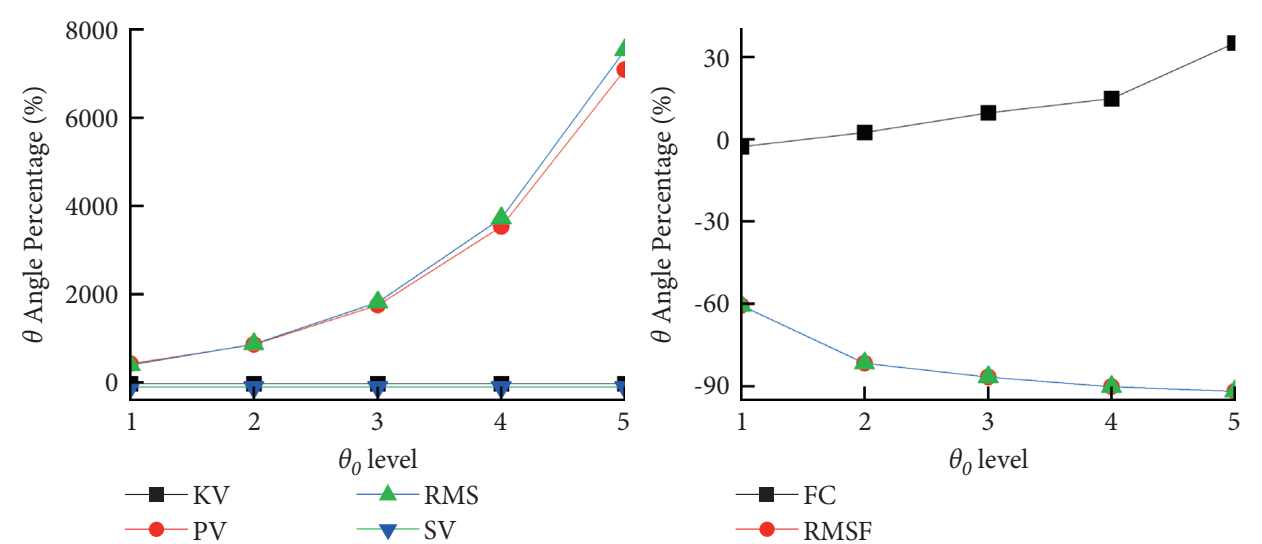

- RMSF

$\triangle-\mathrm{RVF}$
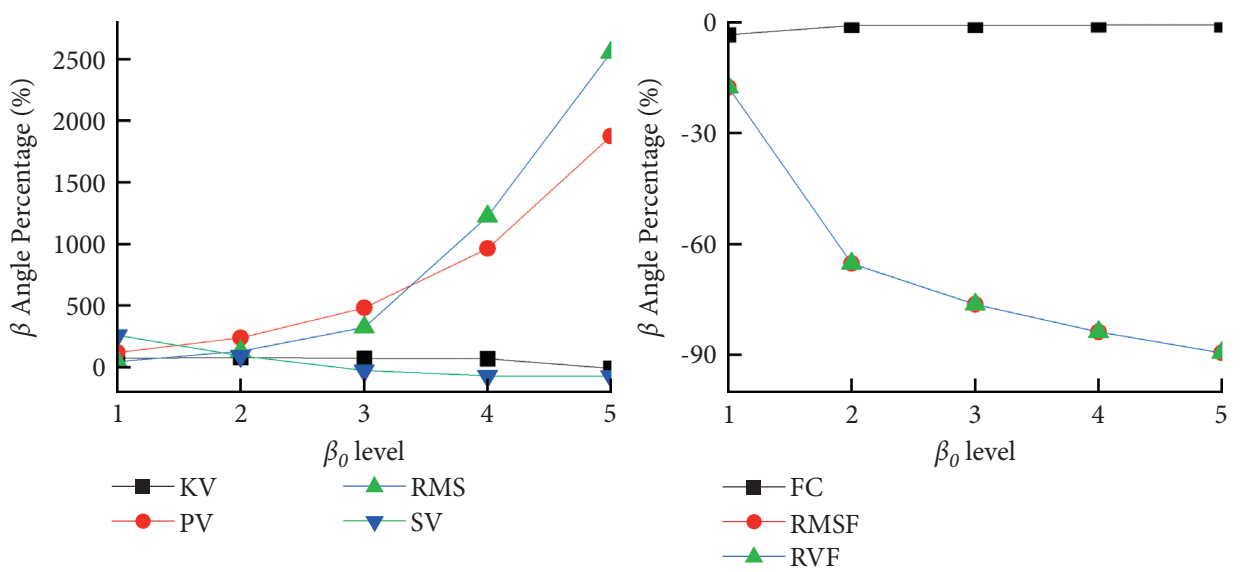

FIGURE 17: Effect of the initial angles on the lift-slewing angle.

\section{Conclusion}

The analysis above indicates that the mass has little effect on the spatial swing angle in the lifting-luffing coupling motion. For the lifting acceleration, the value range that makes the swing angle stable is from $0.004 \mathrm{~m} / \mathrm{s}^{2}$ to $0.01 \mathrm{~m} /$ $s^{2}$. Under the same lifting height, the swing angle fluctuation intensity in the lifting-luffing condition increases with the increase of the tower crane height. PV and RMS can reflect changes in the swing angle state. SV of angle $\beta$ is more sensitive to changes than SV of angle $\theta$. Moreover, frequency-domain and time-domain indicators are sensitive to the swing angle response change when the initial angle varies under the lifting-luffing working condition. The lifting acceleration, the luffing acceleration, and the rope length have more influence on the $\theta$ angle than angle $\beta$. The initial angle has similar effects on angle $\theta$ and angle $\beta$. PV, RMS, RMSF, and RVF present the best sensitivity to changes in the tower crane's spatial swing angle response state in the LLCM.

The mass has little effect on the spatial swing angle in the lifting-slewing coupling motion. For the lifting acceleration, the value range that makes the swing angle stable is $0.004 \mathrm{~m} / \mathrm{s}^{2}$ to $0.01 \mathrm{~m} / \mathrm{s}^{2}$. The dynamic response of angles $\theta$ and $\beta$ diverges from $0.016 \mathrm{~m} / \mathrm{s}^{2}$. When the rope length is between $1 \mathrm{~m}$ and $2 \mathrm{~m}$, the swing angle fluctuates greatly in the lifting-slewing condition. Furthermore, when initial angle $\theta_{0}$ is smaller than initial angle $\beta_{0}$, the angle $\beta$ is not divergent. PV and RMS can reflect changes in the swing angle state, but the PV sensitivity is lower than RMS. The lifting acceleration, the slewing acceleration, the rope length, and the initial angle have more influence on the $\beta$ angle than the $\theta$ angle. PV, RMS, RMSF, and RVF are sensitive to changes in the tower crane's spatial swing angle response state in the LSCM.

According to the parameters of the tower crane in the LSCM and LLCM, the swing angle changes are simulated. The PV, RMS, RMSF, and RVF can be utilized in the controller design. It is conducive to the controllers suppressing the change of the spatial swing angle.

In future work, how is suppressed vibration of tower crane under compound working condition based on the modelings? Which factors do have an effect on fatigue life of tower crane? If do those, the tower crane system will be stable so that it can be used with more safety. 


\section{Appendix}

The elements $B_{i}$ are as follows:

$$
\begin{aligned}
& B_{1}=m w L_{y} \cos \theta+\left(\frac{1}{2}\right) m g L_{y} \cos \beta \sin 2 \theta+m e L_{y} \sin \theta \sin \beta, \\
& B_{2}=\left(\frac{1}{2}\right) m g L_{y} \cos \theta \sin 2 \beta-m e L_{y} \sin \beta \cos \theta, \\
& B_{3}=m e L_{y} \cos \theta \cos \beta+\left(\frac{1}{2}\right) m g L_{y} \cos \beta \sin 2 \theta+m(\dot{k})^{2} R L_{y} \cos \theta+m(\dot{k})^{2}\left(L_{y}\right)^{2} \sin 2 \theta, \\
& B_{4}=\left(\frac{1}{2}\right) m g L_{y} \cos \theta \sin 2 \beta+m e L_{y} \cos \beta \cos \theta+2 m \dot{k} \theta L_{y}^{2} \cos \theta \cos \beta+m w L_{y} R \cos \beta+m w L_{y}^{2} \cos \beta \sin \theta .
\end{aligned}
$$

The elements $A_{i j}$ are as follows:

$$
\begin{aligned}
& A_{11}=m \frac{\left(2 b L_{y}-3 L_{y}^{2}-b^{2}\right)}{3}, \\
& A_{12}=-m L_{y}^{2} \sin \beta \sin \theta, \\
& A_{21}=m \frac{\left(2 b L_{y}-3 L_{y}^{2}-b^{2}\right)}{3}, \\
& A_{21}=-m L_{y}^{2} \sin \beta \sin \theta, \\
& A_{31}=m \frac{\left(2 b L_{y}-3 L_{y}^{2}-b^{2}\right)}{3}, \\
& A_{32}=-m L_{y}^{2} \sin \beta \sin \theta \\
& A_{41}=m \frac{\left(2 b L_{y}-3 L_{y}^{2}-b^{2}\right)}{3}, \\
& A_{42}=-m L_{y}^{2} \sin \beta \sin \theta .
\end{aligned}
$$

\section{Data Availability}

The data used to support the findings of this study are available from the corresponding author upon request.

\section{Conflicts of Interest}

The authors declare that they have no conflicts of interest.

\section{References}

[1] B. Jerman, "An enhanced mathematical model for investigating the dynamic loading of a slewing crane," Proceedings of the Institution of Mechanical Engineers - Part C: Journal of Mechanical Engineering Science, vol. 220, no. 4, pp. 421-433, 2006.

[2] X. G. Yao, Y. B. Feng, L. J. Meng, L. Yao, and H. B. Li, "Influence of structural elasticity on transshipment dynamics of dual-trolley overhead crane," Advances in Mechanical Engineering, vol. 13, no. 7, 2021.

[3] G. Sun and J. Liu, "Dynamic responses of hydraulic crane during luffing motion," Mechanism and Machine Theory, vol. 41, no. 11, pp. 1273-1288, 2006.

[4] B. Jerman and J. Kramar, "A study of the horizontal inertial forces acting on the suspended load of slewing cranes," International Journal of Mechanical Sciences, vol. 50, no. 3, pp. 490-500, 2008.

[5] J. Neupert, E. Arnold, K. Schneider, and O. Sawodny, "Tracking and anti-sway control for boom cranes," Control Engineering Practice, vol. 18, no. 1, pp. 31-44, 2010.

[6] R. Feng, E. Zhang, and M. Dong, "Jib vibration and payload swing of tower cranes in the case of trolley motion," Arabian Journal for Science and Engineering, vol. 46, no. 12, pp. 12179-12191, 2021.

[7] B. Jerman and A. Hribar, "Dynamics of the mathematical pendulum suspended from a moving mass," Tehnicki Vjesniktechnical Gazette, vol. 20, no. 1, pp. 59-64, 2013.

[8] V. K. Augustaitis, V. Gican, A. Jakstas, B. Spruogis, and V. Turla, "Research of lifting equipment dynamics," Journal of Vibroengineering, vol. 16, no. 4, pp. 2082-2088, 2014.

[9] A. A. Shaikh and D. Kumar, "Lifting capacity enhancement of a crawler crane by improving stability," Journal of Theoretical and Applied Mechanics, vol. 54, no. 1, pp. 219-227, 2016.

[10] A. Trabka, "Influence of flexibilities of cranes structural components on load trajectory," Journal of Mechanical Science and Technology, vol. 30, no. 1, pp. 1-14, 2016.

[11] I. Cicek and A. Ertas, "Experimental investigation of beam-tip mass and pendulum system under random excitation," $M e$ chanical Systems and Signal Processing, vol. 16, no. 6, pp. 1059-1072, 2002.

[12] H. Sano, K. Ohishi, T. Kaneko, and H. Mine, "Anti-sway crane control based on dual state observer with sensor-delay correction," in Proceedings of the 2010 Eleventh IEEE International Workshop On Advanced Motion Control (AMC), pp. 679-684, IEEE, Nagaoka, Japan, March 2010.

[13] E. Jarzebowska, A. Urbas, and K. Augustynek, "Analysis of influence of a crane flexible supports, link flexibility, and joint friction on vibration associated with programmed motion execution," Journal of Vibration Engineering \\& Technologies, vol. 8, no. 2, pp. 337-350, 2020.

[14] E. L. de Angelis, "Swing angle estimation for multicopter slung load applications," Aerospace Science and Technology, vol. 89, pp. 264-274, 2019. 
[15] M. Ebrahimi, M. Ghayour, S. M. Madani, and A. Khoobroo, "Swing angle estimation for anti-sway overhead crane control using load cell," International Journal of Control, Automation and Systems, vol. 9, no. 2, pp. 301-309, 2011.

[16] S. M. Fasih, Z. Mohamed, A. R. Husain, L. Ramli, A. M. Abdullahi, and W. Anjum, "Payload swing control of a tower crane using a neural network-based input shaper," Measurement \& Control, vol. 53, no. 7-8, pp. 1171-1182, 2020.

[17] M. H. Fatehi, M. Eghtesad, and R. Amjadifard, "Modelling and control of an overhead crane system with a flexible cable and large swing angle," Journal of Low Frequency Noise, Vibration and Active Control, vol. 33, no. 4, pp. 395-409, 2014.

[18] M. H. Fatehi, M. Eghtesad, D. S. Necsulescu, and A. A. Fatehi, "Tracking control design for a multi-degree underactuated flexible-cable overhead crane system with large swing angle based on singular perturbation method and an energyshaping technique," Journal of Vibration and Control, vol. 25, no. 11, pp. 1752-1767, 2019.

[19] C. Chin, A. H. Nayfeh, and E. Abdel-Rahman, "Nonlinear dynamics of a boom crane," Journal of Vibration and Control, vol. 7, no. 2, pp. 199-220, 2001.

[20] D. C. D. Oguamanam, J. S. Hansen, and G. R. Heppler, "Dynamics of a three-dimensional overhead crane system," Journal of Sound and Vibration, vol. 242, no. 3, pp. 411-426, 2001.

[21] R. M. Ghigliazza and P. Holmes, "Erratum to "On the dynamics of cranes, or spherical pendula with moving supports" [Int. J. Non-Linear Mech. 37 (2002) 1211]," International Journal of Non-Linear Mechanics, vol. 38, no. 2, p. 285, 2003.

[22] F. Ju and Y. S. Choo, "Dynamic analysis of tower cranes," Journal of Engineering Mechanics, vol. 131, no. 1, pp. 88-96, 2005.

[23] V. Gasic, N. Zrnic, and M. Rakin, "Consideration of a moving mass effect on dynamic behaviourof a jib crane structure," Tehnicki Vjesnik-technical Gazette, vol. 19, no. 1, pp. 115-121, 2012.

[24] L. A. Tuan and S.-G. Lee, "Modeling and advanced sliding mode controls of crawler cranes considering wire rope elasticity and complicated operations," Mechanical Systems and Signal Processing, vol. 103, pp. 250-263, 2018.

[25] H. Liu, W. Cheng, and Y. Li, "Dynamic responses of an overhead crane's beam subjected to a moving trolley with a pendulum payload," Shock and Vibration, vol. 2019, Article ID 1291652, 14 pages, 2019.

[26] I. Marinovic, D. Sprecic, and B. Jerman, "A slewing crane payload dynamics," Tehnicki Vjesnik-technical Gazette, vol. 19, no. 4, pp. 907-916, 2012.

[27] T.-S. Wu, M. Karkoub, H. Wang, H.-S. Chen, and T.-H. Chen, "Robust tracking control of MIMO underactuated nonlinear systems with dead-zone band and delayed uncertainty using an adaptive fuzzy control," IEEE Transactions on Fuzzy Systems, vol. 25, no. 4, pp. 905-918, 2017.

[28] S. Otto and R. Seifried, "Real-time trajectory control of an overhead crane using servo-constraints," Multibody System Dynamics, vol. 42, no. 1, pp. 1-17, 2018.

[29] N. Sun, Y. Fu, T. Yang, J. Zhang, Y. Fang, and X. Xin, "Nonlinear motion control of complicated dual rotary crane systems without velocity feedback: design, analysis, and hardware experiments," IEEE Transactions on Automation Science and Engineering, vol. 17, no. 2, pp. 1017-1029, 2020.

[30] R. Miranda-Colorado, "Robust observer-based anti-swing control of 2D-crane systems with load hoisting-lowering," Nonlinear Dynamics, vol. 104, no. 4, pp. 3581-3596, 2021.
[31] J. Wang, J. Yang, Y. Bai, Y. Zhao, Y. He, and D. Yao, "A comparative study of the vibration characteristics of railway vehicle axlebox bearings with inner/outer race faults," Proceedings of the Institution of Mechanical Engineers - Part F: Journal of Rail and Rapid Transit, vol. 235, no. 8, pp. 1035-1047, 2021. 